University of South Carolina

Scholar Commons

2014

\title{
Tuning the Underwater Oleophobicity of Graphene Oxide Coatings via UV Irradiation
}

H. Li

Yi Huang

huangyi@mailbox.sc.edu

Y. Mao

W. Xu

Harry J. Ploehn

University of South Carolina - Columbia, ploehn@cec.sc.edu

See next page for additional authors

Follow this and additional works at: https://scholarcommons.sc.edu/eche_facpub

Part of the Chemical Engineering Commons, and the Chemistry Commons

\section{Publication Info}

Published in Chemical Communications, Volume 50, Issue 69, 2014, pages 9849-9851.

(c) Chemical Communications 2014, Royal Society of Chemistry

Li, H., Huang, Y., Mao, Y., Xu, W. L., Ploehn, H. J., \& Yu, M. (2014). Tuning the underwater oleophobicity of graphene oxide coatings via UV irradiation. Chemical Communications, 50(69), 9849-9851.

http://dx.doi.org/10.1039/C4CC03940H

This Article is brought to you by the Chemical Engineering, Department of at Scholar Commons. It has been accepted for inclusion in Faculty Publications by an authorized administrator of Scholar Commons. For more information, please contact digres@mailbox.sc.edu. 


\section{Author(s)}

H. Li, Yi Huang, Y. Mao, W. Xu, Harry J. Ploehn, and Miao Yu 


\section{ChemComm}

Cite this: Chem. Commun., 2014 50,9849

Received 22nd May 2014,

Accepted 8th July 2014

DOI: $10.1039 / \mathrm{c} 4 \mathrm{cc} 03940 \mathrm{~h}$

www.rsc.org/chemcomm

\section{Tuning the underwater oleophobicity of graphene oxide coatings via UV irradiation $\dagger$}

\author{
Hang Li, ${ }^{a b}$ Yi Huang, ${ }^{a b}$ Yating Mao, ${ }^{a}$ Weiwei L. Xu, ${ }^{a b}$ Harry J. Ploehn ${ }^{a}$ and \\ Miao Yu*ab
}

Ultraviolet (UV) irradiation was utilized to gradually modify the chemistry and structure of graphene oxide (GO) flakes, as confirmed by XPS and AFM. Ultrathin GO coatings/membranes, made of UV-irradiated flakes, showed tunable underwater oleophobicity. UV-treated, superoleophobic GO membranes exhibited excellent antifouling capability for oil/water separation.

Surfaces with controllable underwater oil-adhesion have attracted great attention due to their potential applications in oil/water separation, oil-repellent materials, microfluidic devices, anti-bioadhesion materials, and robust antifouling materials. ${ }^{1-4}$ Fish scales are well known to possess the underwater superoleophobic/low oil adhesive properties. Studies on fish scales have shown that a hydrophilic mucus layer and micro/nanoscale surface roughness are essential for their superior performance. ${ }^{5}$ Consistently, the underwater oil wettability of a solid surface has been found to depend strongly on the surface chemical composition and roughness. ${ }^{5,6}$ Graphene oxide (GO) is a well-known hydrophilic material due to its unique chemistry. ${ }^{7}$ Oxygen-containing functional groups, such as carboxyl, carbonyl, hydroxyl and epoxy, are distributed at edges and structural defects of GO flakes. Therefore, GO flakes with fine-tuned chemistry and roughness are promising materials for fabricating surfaces with desired underwater oleophobicity.

Oxidative etching has been proven to be an effective way to create or enlarge structural defects on graphene-based materials. ${ }^{8,9}$ Generated defects increased the nano-scale roughness of the single GO flakes. ${ }^{10}$ In addition, oxidative etching also improved the hydrophilicity of GO, probably resulting from the introduced oxygen groups around the expanded and/or newly generated defects. ${ }^{7,11}$ Oxidative etching, therefore, seems to be a viable way of controlling the underwater oleophobicity of GO by modifying GO

\footnotetext{
${ }^{a}$ Department of Chemical Engineering, University of South Carolina, Columbia, SC 29208, USA. E-mail: yumiao@cec.sc.edu

${ }^{b}$ Catalysis for Renewable Fuels Center, University of South Carolina, Columbia, SC 29208, USA

$\dagger$ Electronic supplementary information (ESI) available: Experimental section and Fig. S1-S3. See DOI: 10.1039/c4cc03940h
}

morphology and hydrophilicity. However, the precise control of the hydrophilicity/underwater oleophobicity of GO via oxidative etching has not been reported. One potential reason could be that etching reaction occurs in the oxidative gas phase, which usually proceeds fast and is difficult to control. ${ }^{8,9}$ Also, only single or few-layered graphene-based suspended flakes or coatings, instead of powder, have been etched uniformly in the gas phase, ${ }^{8,10}$ which may limit their large-scale productivity for potential applications. Here, we report the novel use of ultraviolet (UV) light to controllably modify the chemistry and structure of GO flakes in aqueous media. UV irradiation has been proved to be capable of either reducing or oxidizing graphene-based materials, depending on the reducing or oxidative nature of the surrounding environment. ${ }^{12-18}$ We demonstrate that by simply controlling the UV etching time for dispersed GO flakes in water, the resulting GO coatings can be converted into underwater superoleophobic coatings. In addition, this method is very promising for large-scale production. The probable mechanism of UV oxidative etching can be that ozone molecules, generated by UV activation, adsorb onto the local pristine graphene region on GO and react with its surface to form oxygen-containing groups and create defects by breaking $\mathrm{C}-\mathrm{C}$ bonds, as suggested by previous studies. ${ }^{15-17}$ Cyclic oil/water separation tests of the UV-treated, superoleophobic GO coatings/membranes exhibited excellent antifouling and ease-of-cleaning performance. Such an effective and facile methodology to modify the chemistry and morphology of GO flakes may provide great opportunities to generate functional coatings/surfaces with drastically improved underwater oil repellent properties.

We prepared GO by an improved Hummers' method. ${ }^{19}$ Dry GO powder was then well dispersed in deionized (DI) water by ultrasonication. After centrifugation, GO agglomerates were removed. The final GO flakes are $\sim 1000 \mathrm{~nm}$ in size (Fig. 1a) and single-layered (Fig. 1b), as confirmed by atomic force microscopy (AFM). We then diluted the GO dispersion to a concentration of $0.0625 \mathrm{mg} \mathrm{mL}^{-1}$. A UV lamp was applied as the light source to conduct the etching treatment of the GO dispersion for different times, from 0 to $90 \mathrm{~min}$ (labelled as 0-GO, 10-GO, 30-GO, 60-GO and 90-GO for convenience). UV treatment in water, instead of in air, provides better control of the 

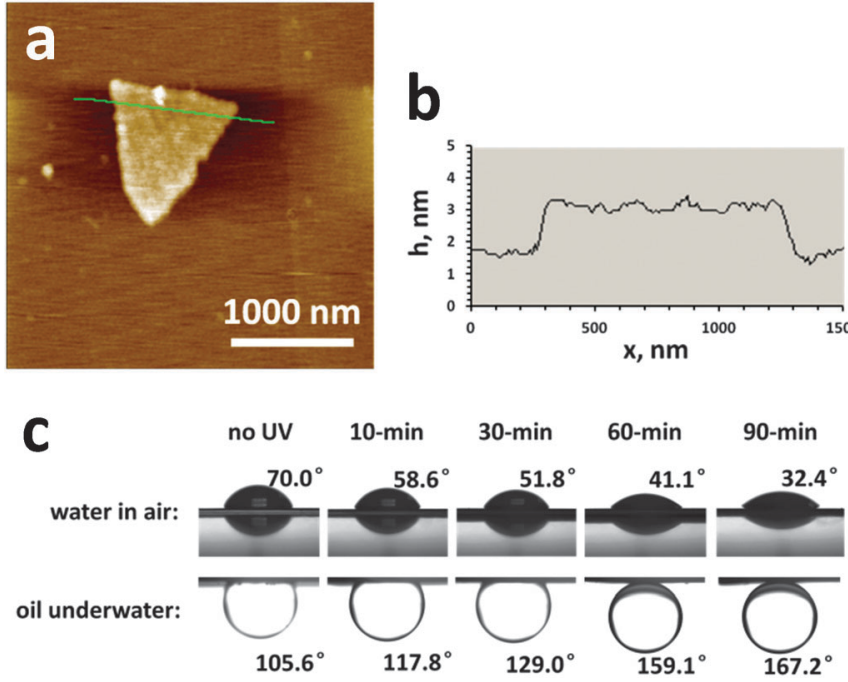

Fig. 1 (a) An AFM image of a GO flake on freshly cleaved mica. (b) The height profile across the green line in (a). $h$, height; $x$, position. (c) The water contact angle in air and the hexadecane (HD) contact angle in water for GO membranes with different UV treatment times.

GO etching process. This is because good dispersion of GO in water and vigorous stirring ensure uniform UV irradiation of GO flakes. The GO dispersion was then used to fabricate $10 \mathrm{~nm}$ coatings/membranes onto flat anodic aluminium oxide (AAO) supports (20 $\mathrm{nm}$ pore size) by a vacuum filtration method, following a similar procedure to our previous work. ${ }^{20}$ Water contact angle in air and oil contact angle in water measurements were subsequently performed for the GO coatings/membranes (see ESI $\dagger$ for experimental details). As shown in Fig. 1c, the water contact angle in air decreases gradually with the increase of UV treatment time, from $70.0^{\circ}$ for the 0-GO membrane to $32.4^{\circ}$ for the $90-\mathrm{GO}$ membrane. From the underwater hexadecane (HD) contact angle measurements, a slight contact angle increase from 0-GO to 30-GO could be noticed. Surprisingly, we found that when UV treatment time increased to $60 \mathrm{~min}$, the underwater oil contact angle became $159.1^{\circ}$, which is considered to be superoleophobic $\left(>150^{\circ}\right) \cdot{ }^{21}$ The 90 -GO membrane shows an even higher oil contact angle $\left(167.2^{\circ}\right)$ than the 60 -GO membrane. These results indicate that the oil-adhesion characteristics of an oil-water-solid triple-phase system could be tuned by changing UV exposure time (see ESI $\dagger$ for details). The UV-treated GO flakes are stable in air, as suggested by Raman spectra shown in Fig. S2 (see ESI $\dagger$ for details).

To explain the underlying mechanism, we further conducted X-ray photoelectron spectroscopy (XPS) and Raman spectroscopy measurements for GO dispersion after different UV treatment times. After deconvoluting the overlapping XPS peaks of C_1s (see Fig. S1, ESI, $\dagger$ for C_1s XPS spectra), the percentage of carbon in different chemical environments can be obtained, as shown in Fig. 2a. It is seen that as the UV treatment time increased, the percentage of oxidized carbon (including $\mathrm{C}-\mathrm{O}, \mathrm{C}=\mathrm{O}$ and $\mathrm{COOH}$ ) increased. This is because the percentage of $\mathrm{C}$ in $\mathrm{C}=\mathrm{O}$ and $\mathrm{COOH}$ increased, whereas that of $\mathrm{C}-\mathrm{O}$ didn't change much. These groups on GO have been proved to have strong affinity to water molecules, $^{22}$ and thus would help form a thin layer of water barrier to lower the oil adhesion. A higher percentage of hydrophilic
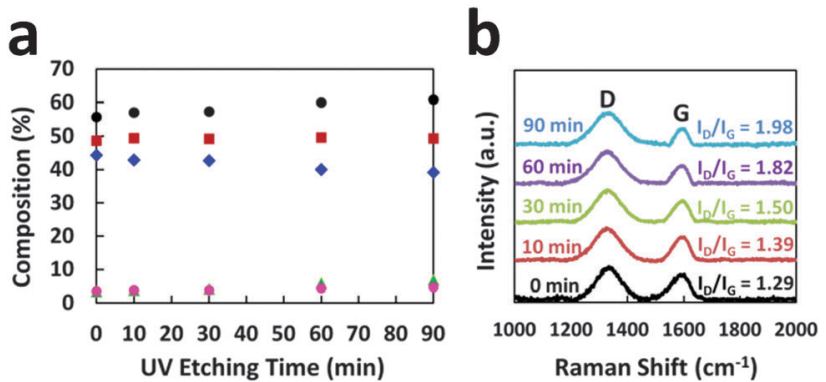

Fig. 2 (a) Percentage of differently bonded carbon on GO analyzed by XPS: total oxidized carbon; total unoxidized carbon; $\mathrm{C}-\mathrm{O}$; $\mathrm{C}=\mathrm{O}$; and $-\mathrm{COOH}$; (b) Raman spectra of $\mathrm{GO}$ after different UV treatment times from 0 to $90 \mathrm{~min}$

oxygen-containing functional groups after longer UV treatment time, therefore, may contribute to the better wettability of water in air and lower underwater oil-adhesion. Raman spectra (Fig. 2b) show that the $I_{\mathrm{D}} / I_{\mathrm{G}}$ ratio increased as the UV treatment time increased, suggesting higher disorder of the planar structure of the GO flakes. This may be caused by the enlarged or newly generated structural defects. ${ }^{8}$ We then deposited thin coatings onto mica using GO dispersions with different UV exposure times and directly conducted AFM on them, as shown in Fig. 3a-d. We can see that for the 0-GO coating, the surface is fairly flat and continuous. The 30-GO coating shows a surface decorated with defects from $\sim 80$ to $120 \mathrm{~nm}$ in size, while the 60-GO coating exhibits a very disordered surface with large holes from $\sim 300$ to $500 \mathrm{~nm}$. The 90-GO coating seems more like isolated islands, apparently due to over-etching. ${ }^{9,10}$ The generated defects eventually increased the nanoscale surface roughness of the GO coatings, as indicated by the height profiles. Therefore, we conclude that both the chemical composition and structural changes after UV oxidative etching lead to the drastically improved underwater oleophobicity of GO coatings (see ESI $\dagger$ for details).

As is known, fouling of nano/ultrafiltration membranes in oil/water separation is a longstanding issue and a major economic barrier for their wide range of applications. ${ }^{23}$ Membranes with underwater superoleophobic surfaces have great potential to realize antifouling in oily wastewater treatment. ${ }^{24}$ In order to utilize such excellent underwater superoleophobicity of our UV-treated GO, we prepared $10 \mathrm{~nm}$ GO membranes on polyamide (PA) supports using UV-treated GO (0, 30 and 60-GO) and conducted a series of oil emulsion filtration procedures in a dead-end system to investigate their antifouling performance. 1500 ppm HD-in-water emulsion, stabilized by 100 ppm sodium dodecyl sulfate (SDS) after 1 hour of sonication, was used as the feed. The filtration process contains three cycles. In each individual cycle, pure water filtration was performed, followed by emulsion separation. During the interval between two neighboring cycles, a simple water flush cleaning process was applied to clean the membrane surface. Therefore, the recovery of pure water flux in each cycle could be an indicator of the membrane fouling degree. The 60 -GO membrane shows $\sim 100 \%$ pure water recovery for all cycles (Fig. 4), suggesting superior antifouling performance. In stark contrast, the 0-GO membrane (Fig. S3a, ESI $\dagger$ ) exhibits severe membrane fouling, since the pure 

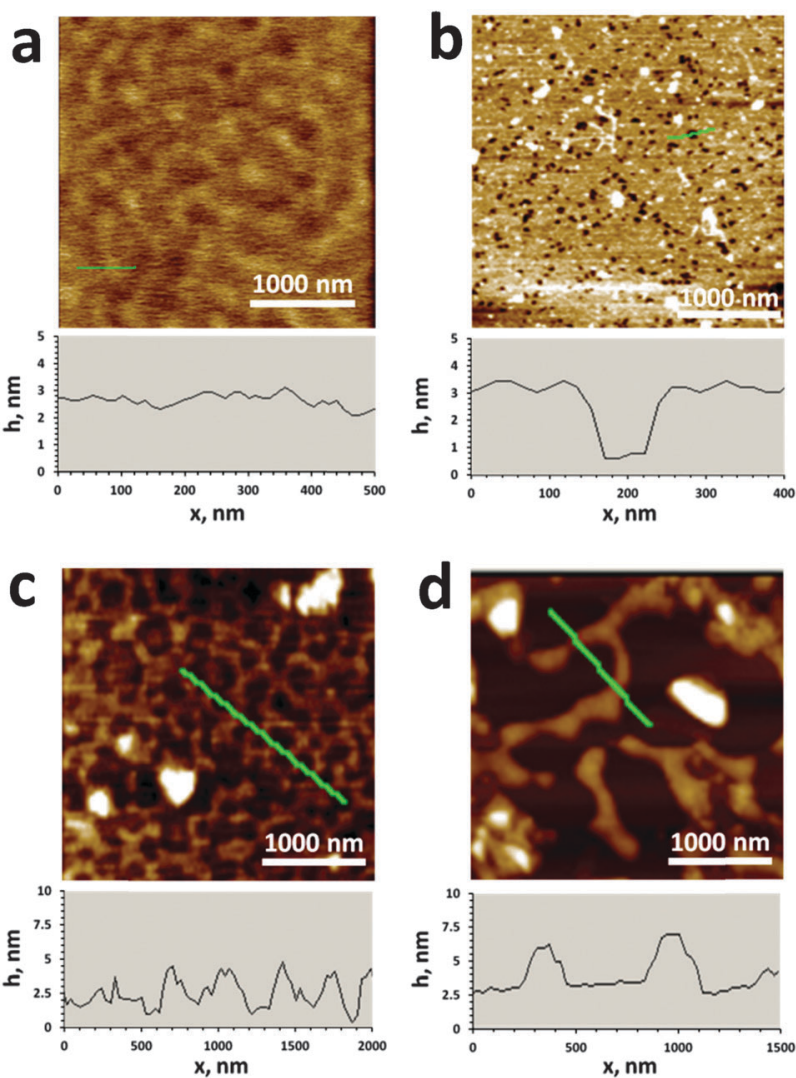

Fig. 3 AFM images and height profiles across the green lines for (a) 0-GO coating, (b) 30-GO coating, (c) 60-GO coating, and (d) 90-GO coating on mica. $h$, height; $x$, position.

water flux recovery for the 2nd cycle is only $51.7 \%$ and further decreases for the 3rd cycle. The 30-GO membrane (Fig. S3b, ESI $\dagger$ ) shows improved antifouling performance with a 2nd cycle pure water flux recovery of $90.1 \%$. This again validates our methodology of utilizing UV irradiation to tune the chemical composition and structure of GO flakes to realize a low oil-adhesion, underwater superoleophobic surface. The oil rejection for the tested membranes

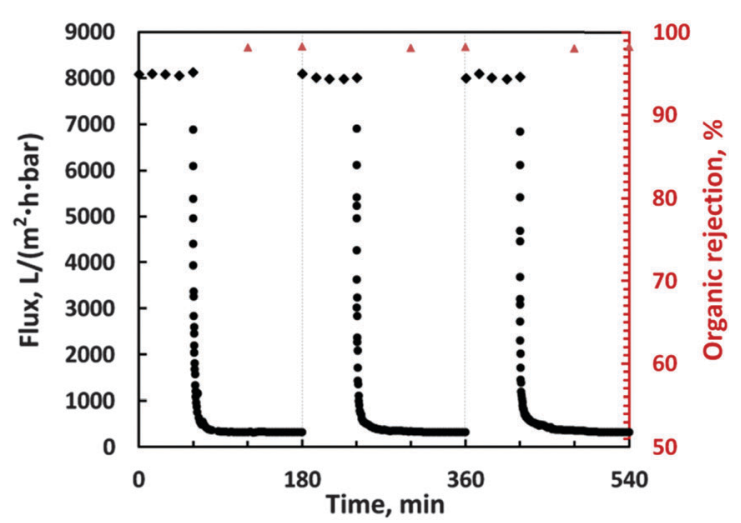

Fig. 4 cyclic water/oil separation test for a $10 \mathrm{~nm}$ 60-GO membrane on the PA support. ( $)$ total flux in oil-in-water emulsion separation. ( $\bullet$ ) pure water flux. ( $\mathbf{\Lambda})$ total organic rejection. was all around 98.0\% (see ESI $\dagger$ for a detailed experimental setup and discussion).

In summary, UV-irradiation was utilized as an effective and facile approach to tune the underwater oleophobicity of GO coatings/membranes by gradually modifying $\mathrm{GO}$ flake composition and surface morphology. Superoleophobic GO membranes, made of UV-treated GO flakes, showed excellent antifouling capability and greatly improved oil emulsion separation performance. We expect that this facile strategy to tune the underwater oleophobicity of GO may help design novel graphene-based materials/coatings for a wide range of applications in oil contaminated environments.

We acknowledge the financial support from USC start-up funding and the USC SPARC graduate student grant. We also thank Drs Shuguo Ma, Qian Wang and Xinyu Huang for their experimental assistance with XPS, contact angle and Raman measurements, respectively.

\section{Notes and references}

1 A. K. Geim, S. V. Dubonos, I. V. Grigorieva, K. S. Novoselov, A. A. Zhukov and S. Y. Shapoval, Nat. Mater., 2003, 2, 461-463.

2 Y. Huang, M. Liu, J. Wang, J. Zhou, L. Wang, Y. Song and L. Jiang, Adv. Funct. Mater., 2011, 21, 4436-4441.

3 D. Tian, Z. Guo, Y. Wang, W. Li, X. Zhang, J. Zhai and L. Jiang, Adv. Funct. Mater., 2013, 24, 536-542.

4 M. Liu, X. Liu, C. Ding, Z. Wei, Y. Zhu and L. Jiang, Soft Matter, 2011, 7, 4163-4165.

5 R. Blossey, Nat. Mater., 2003, 2, 301-306.

6 T. Sun, G. Qing, B. Su and L. Jiang, Chem. Soc. Rev., 2011, 40, 2909-2921.

7 D. R. Dreyer, S. Park, C. W. Bielawski and R. S. Ruoff, Chem. Soc. Rev., 2010, 39, 228-240.

8 S. P. Koenig, L. Wang, J. Pellegrino and J. S. Bunch, Nat. Nanotechnol., 2012, 7, 728-732.

9 Z. Zeng, X. Huang, Z. Yin, H. Li, Y. Chen, H. Li, Q. Zhang, J. Ma, F. Boey and H. Zhang, Adv. Mater., 2012, 24, 4138-4142.

10 T. H. Han, Y.-K. Huang, A. T. L. Tan, V. P. Dravid and J. Huang, J. Am. Chem. Soc., 2011, 133, 15264-15267.

11 A. Bagri, C. Mattevi, M. Acik, Y. J. Chabal, M. Chhowalla and V. B. Shenoy, Nat. Chem., 2010, 2, 581-587.

12 S. B. Bon, M. Piccinini, A. Mariani, J. M. Kenny and L. Valentini, Diamond Relat. Mater., 2011, 20, 871-874.

13 K. S. Subrahmanyam, P. Kumar, A. Nag and C. N. R. Rao, Solid State Commun., 2010, 150, 1774-1777.

14 P. Fabbri, L. Valentini, S. Bittolo Bon, D. Foix, L. Pasquali, M. Montecchi and M. Sangermano, Polymer, 2012, 53, 6039-6044.

15 S. Huh, J. Park, Y. S. Kim, K. S. Kim, B. H. Hong and J.-M. Nam, ACS Nano, 2011, 5, 9799-9806.

16 Y. C. Cheng, T. P. Kaloni, Z. Y. Zhu and U. Schwingenschlögl, Appl. Phys. Lett., 2012, 101, 073110.

17 Z. Shichao, P. S. Sumedh, L. Zhiting and L. Haitao, Nanotechnology, 2012, 23, 355703.

18 N. Mitoma, R. Nouchi and K. Tanigaki, J. Phys. Chem. C, 2013, 117, 1453-1456.

19 D. C. Marcano, D. V. Kosynkin, J. M. Berlin, A. Sinitskii, Z. Sun, A. Slesarev, L. B. Alemany, W. Lu and J. M. Tour, ACS Nano, 2010, 4, 4806-4814.

20 H. Li, Z. Song, X. Zhang, Y. Huang, S. Li, Y. Mao, H. J. Ploehn, Y. Bao and M. Yu, Science, 2013, 342, 95-98.

21 A. K. Kota, G. Kwon, W. Choi, J. M. Mabry and A. Tuteja, Nat. Commun., 2012, 3, 1025.

22 R. R. Nair, H. A. Wu, P. N. Jayaram, I. V. Grigorieva and A. K. Geim, Science, 2012, 335, 442-444.

23 A. Asatekin and A. M. Mayes, Environ. Sci. Technol., 2009, 43, 4487-4492.

24 L. Zhang, Z. Zhang and P. Wang, NPG Asia Mater., 2012, 4, e8. 